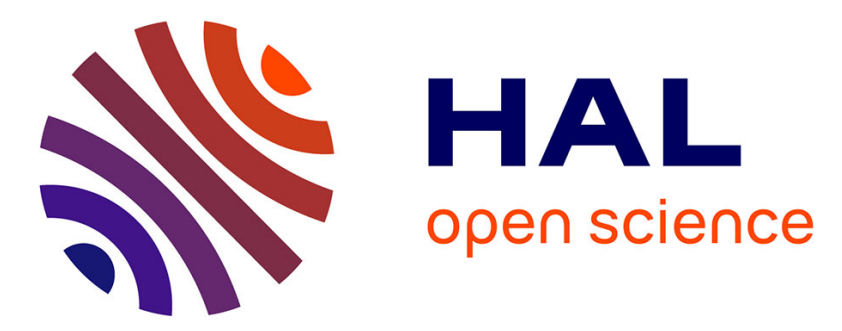

\title{
International Environmental Agreements: the case of costly monetary transfers
}

Basak Bayramoglu, Jean-François Jacques

\section{To cite this version:}

Basak Bayramoglu, Jean-François Jacques. International Environmental Agreements: the case of costly monetary transfers. Environmental and Resource Economics, 2015, 62 (4), pp.745-767. 10.1007/s10640-014-9837-1 . hal-01511386

\section{HAL Id: hal-01511386 \\ https://hal.science/hal-01511386}

Submitted on 20 Apr 2017

HAL is a multi-disciplinary open access archive for the deposit and dissemination of scientific research documents, whether they are published or not. The documents may come from teaching and research institutions in France or abroad, or from public or private research centers.
L'archive ouverte pluridisciplinaire HAL, est destinée au dépôt et à la diffusion de documents scientifiques de niveau recherche, publiés ou non, émanant des établissements d'enseignement et de recherche français ou étrangers, des laboratoires publics ou privés.

\section{다(1)(2)}

Distributed under a Creative Commons Attribution - ShareAlikel 4.0 International 


\title{
International Environmental Agreements: The Case of Costly Monetary Transfers
}

\author{
Basak BAYRAMOGLU\&Jean-François JACQUES ${ }^{\ddagger}$
}

September 10, 2014

\begin{abstract}
Most existing international environmental agreements to resolve transboundary pollution problems appear constrained in the sense that either monetary transfers accompany uniform abatement standards (agreements based on a uniform standard with monetary transfers), or differentiated abatement standards are established, but without monetary transfers (agreements based on differentiated standards). For two asymmetric countries facing the challenge of a transboundary pollution problem, we compare the relative efficiency of these two second-best agreements. We study especially the role of the costs associated with transfer payments across countries in the choice of these agreements. To conduct this analysis, we use a negotiation game and the generalized Nash bargaining solution (Nash, 1953) as the equilibrium. For total welfare, our findings show that countries collectively
\end{abstract}

*INRA-AgroParisTech, UMR 210 Economie Publique, F-78850 Thiverval-Grignon, France. Tel: (0)1 440872 77, E-mail: Basak.Bayramoglu@grignon.inra.fr.

${ }^{\dagger}$ LEDa-CGEMP, Université Paris-Dauphine, Place du maréchal de Lattre de Tassigny, 75775 Paris Cedex 16. CRIEF, Université de Poitiers, 2 rue Jean Carbonnier Bat 1, BP(623) , 86022 Poitiers Cedex. E-mail: jacques@dauphine.fr.

${ }^{\ddagger}$ We are grateful to Bernard Caillaud for the motivation of the paper, and to Ivar Ekeland for his invitation to the University British Columbia (UBC) where the paper was written. We would like to thank Brian Copeland, Philippe Bernard, Françoise Forges and two referees for their constructive remarks. We would also like to thank the participants of the EURiSCO seminar at Université Paris Dauphine, Paris (June 2006), the Third World Congress of Environmental and Resource Economists, Kyoto (July 2006), and the PGPPE Workshop, Marseille (April 2007). The authors are responsible for all the remaining errors. 
prefer the uniform to the differentiated agreement if the cost of transfers is sufficiently low compared to the ratio for countries of the difference of the abatement costs between the two agreements. In the analysis of individual welfare, we also discuss the reluctance of one country to sign a specific type of agreement even if it is better off than in the case of non-cooperation.

Keywords: transboundary pollution, cooperative games, bargaining, standards, transfers.

$J E L:$ Q50, C71.

\section{Introduction}

The first commitment period of the Kyoto Protocol on Climate Change (1997) expired in 2012 and, at the international level, discussions have already begun concerning the design of a post-Kyoto protocol. Recent international negotiations in Doha in 2012 and in Warsaw in 2013 resulted in some progress; however, the design of a future agreement on climate change is still unknown. The design of International Environmental Agreements (IEAs) is crucial in order to provide the right incentives for countries to participate in the agreement and to comply with its provisions in the future.

Different types of IEAs have been signed by countries in the past, to resolve transboundary pollution problems. These agreements can be distinguished by the type of abatement standards (uniform or differentiated) and by the existence or not of compensatory transfers among countries. Most prior agreements appear constrained in the sense that not all available tools are used for their design. That is, uniform abatement targets can be associated with monetary transfers while differentiated abatement targets cannot. In this paper, for two asymmetric countries facing the challenge of a transboundary pollution problem, we compare the relative efficiency of two second-best agreements frequently observed in reality based on uniform versus differentiated abatement standards.

In particular, we study the role of the costs associated with transfer payments across countries in the choice of these agreements. It can be costly to implement monetary transfers across countries in that these transfers could be qualified as 'imperfect'. There can be losses in transfer payments across countries, meaning that the amount given is greater than the amount received. Two arguments could be put forward to explain the existence of 
imperfect transfers. The first argument is that there are administrative costs involved in donor countries to collect transfers and to deliver them to recipient countries. The second argument is the existence of a 'political' cost: it can be costly, in political terms, for the donor country's government to convince electors and national lobbies of the necessity for transfer payments to another country.

We compare the relative efficiency of two second-best agreements: an agreement based on a uniform standard with monetary transfers (hereafter denoted UT) and an agreement based on differentiated standards without transfers (hereafter denoted D). ${ }^{1}$ For heterogeneous countries, both types of agreements can lead to second-best equilibria. The associated constraints are very different in nature. The UT agreement imposes the constraint of uniformity of abatement standards, but allows for side payments across countries. Conversely, a D agreement could lead to cost-efficiency by exploiting the heterogeneity of countries via differentiated reduction rates in emissions. However, as side payments are absent, this type of agreement may imply asymmetric distribution of the gains from cooperation to the extent that some countries may prefer not to participate in a D agreement. For example, the Oslo Protocol on Further Reduction of Sulphur Emissions (1994) includes differentiated standards, but does not involve side payments. In this agreement, differentiated standards are represented by different sulphur emissions ceilings (in kt per year) specific to each signatory country in 2010: France, 737 kt; United Kingdom, 980 kt; Bulgaria, 1127 kt, etc.

Despite the fact that uniform standards do not satisfy cost-efficiency for asymmetric countries, we observe frequent use of these rules in IEAs (Hoel, 1991; Finus and Rundshagen, 1998; Harstad, 2007). Potential signatory countries to these agreements typically bargain over uniform emission reductions, whereby countries must reduce their current emissions to achieve a certain percentage of their emissions in a base year. The uniform rule means that this percentage reduction is the same across countries. ${ }^{2}$ The use of uniform standards in this agreement does not allow cost-efficiency to be attained, as these standards do not take account of countries' different characteristics (Hoel, 1992). The Montreal Protocol (1987) on Substances

\footnotetext{
${ }^{1}$ Our aim is to compare the welfare related to these two agreements, not to explain why these arrangements emerge.

${ }^{2}$ The term 'uniform standard' could potentially be misleading. A more appropriate term would be 'equiproportional emissions reductions'; however, for simplicity we use uniform standard to underline the contrast with 'differentiated standards'.
} 
that Deplete the Ozone Layer, which specifies emissions reduction of CFCs and halons by $20 \%$ relative to 1986 emission levels, is an example of a UT agreement (Finus, 2001). This protocol established a side-payment scheme (the Multilateral Fund) in 1990, from developed to less-developed countries to help the latter finance their compliance costs. ${ }^{3}$

In the context of a transboundary pollution problem, this paper examines the following questions: How do heterogeneous countries gain from signing agreements with uniform standards? Do side payments help to make uniform standards preferred, despite their being costly to implement? To address these questions, we compare the relative efficiency of the UT and D agreements, using a negotiation game and the generalized Nash bargaining solution (Nash, 1953) as equilibrium.

In the literature on IEAs, there are two main types of models: membership models and compliance models. Membership models are based on the free-rider incentives for countries not to participate in an IEA. However, these models assume that countries respect the obligations of any agreement they sign. Finus (2008) distinguishes between cooperative and non-cooperative membership models. Cooperative models use the stability concept of the core (Chander and Tulkens, 1995 and 1997) to define a transfer scheme that allows for a stable grand coalition to be profitable for each member. Non-cooperative models use the concept of internal and external stability of coalitions (Barrett, 1994; Carraro and Siniscalco, 1993; Hoel, 1992) to represent the incentives for individual countries' membership, based on individual payoff maximization. The complexity of non-cooperative models of membership usually requires some simplifying assumptions, such as identical countries. In contrast to these models which are aimed at analyzing the drivers of cooperation among countries and to characterize the stable size of a coalition, the objective of our analysis is to compare the 'size of the cake' achieved by alternative (second-best) IEAs. For this analysis, we assume a cooperative spirit in the agreement in the sense of countries wanting to improve their payoffs through a bargaining process. Therefore, we adopt a cooperative game concept ${ }^{4}$ which refers to the generalized Nash bargaining

\footnotetext{
${ }^{3}$ The Stockholm Convention on Persistent Organic Pollutants, signed in 2001, is another example of a UT agreement.

${ }^{4}$ Several papers study environmental problems in a cooperative bargaining framework, such as the Nash bargaining solution: see among others Hoel (1991), Carraro and Siniscalco (1993), and Buchholz et al. (2005). However, to take account of the dynamic process of bargaining, negotiations can be modelled by non-cooperative bargaining games. Work on
} 
solution (Nash, 1953). This solution satisfies various axioms, especially that of Pareto efficiency. More generally, the bargaining outcome will depend on the relative bargaining powers of countries (Jéhiel, 1997).

Maler (1989) is the first paper to show the inefficiency of uniform abatement standards in IEAs. Maler (1989) suggests that the protocol to the 'Convention on Long Range Transboundary Air Pollution' (1985), based on a uniform standard, implies insufficient emission reductions compared to the $40 \%$ average abatement associated with the cooperative solution. The inefficiency of uniform abatement standards is stressed also by Newbery (1990). Hoel (2002) mentions two drawbacks to agreements based on uniform standards: absence of cost-efficiency, and dissatisfaction for some countries of the parti cipation constraint (i.e., some countries might prefer not to cooperate). The literature provides a number of arguments related to the use of uniform standards in IEAs: informational problems (Larson and Tobey, 1994; Harstad, 2007), 'focal points' (Eyckmans, 1999; Brandt, 2004), stability of IEAs (Finus, 2008), and international trade (Copeland and Taylor, 2005). Brandt (2004) proposes a model with asymmetric information which shows that the lowest uniform emission reduction allows the participation constraint to be satisfied. Finus (2008) indicates that the use of uniform quotas, including a common quota, a median quota, or the lowest quota proposal, increases the stability of coalitions in the framework of the empirical Stability of Coalitions (STACO) model. In an experimental setting, Dannenberg et al. (2010) show that an IEA based on a uniform quota as the lowest common denominator, is able to increase both the coalition size and average abatement efforts. Also, monetary transfers have been shown to increase the stability of coalitions for asymmetric countries (Barrett, 2001; Fuentes-Albero and Rubio, 2010; Weikard, 2009). However, these papers have not taken into account the costs associated with transfer payments and their impact on aggregate welfare.

We follow the literature on the relative efficiency of second-best agreements. Endres (1997) compares the total welfare for two asymmetric countries under two different types of IEAs: a quota agreement and a tax agreement. The lowest common denominator is used as a rule for choosing between countries' proposals, a principle that follows the assumption of absence of compensatory payments. Finus and Rundshagen (1998) compare the same agreements as Endres (1997), but in a dynamic context using renegotiation-

environmental problems includes, among others, Chen (1997), Compte and Jéhiel (1997), Caparros et al. (2004), and Courtois and Tazdait (2011). 
proof equilibria which allow for credible threats of punishment. Bayramoglu and Jacques (2011) study, for similar countries, the role of fixed costs in abatement technology for the relative efficiency of two second-best agreements: an agreement based on a uniform standard without transfers and an agreement based on differentiated standards with transfers. In our paper, we adopt a static framework and do not take account of the fixed costs in abatement technology. The design of the agreements we consider also differs. We study an agreement based on a uniform standard with transfers, and an agreement based on differentiated standards without transfers. The negotiated levels of quotas and transfers are endogenously determined in a negotiation game model, where it is assumed that, if negotiations break down, countries obtain their payoffs at the Nash equilibrium. More importantly, we model the imperfection of transfers in order to investigate the role of costly monetary transfers for the outcome of international environmental negotiations.

In a simple negotiation game model under perfect information, we show that the agreement based on a uniform standard with transfers could outperform, in terms of total and individual welfare, the agreement based on differentiated standards without transfers. This analysis is carried out for a concave benefit function from global abatement and a convex abatement cost function. ${ }^{5}$ Heterogeneous countries might prefer to sign a uniform rather than a differentiated agreement, even though the transfer payments across countries in the uniform agreement imply costs for the donor country. The explanation can be summed up as follows: if it is less expensive in transfer costs to give incentives to the country with weak incentives to abate at a lower cost, then it is in the interests of both countries to sign the uniform agreement. To our knowledge, no other studies employ the argument of imperfect transfers across countries in IEAs.

The paper is organized as follows. Section 2 presents the model. This corresponds to a description of the threat point of the negotiations, and defines the bargaining-efficient outcomes for the UT and D agreements. Section 3 and Section 4 present total and individual welfare comparisons respectively between the two agreements. Section 5 offers some concluding remarks.

\footnotetext{
${ }^{5}$ Bayramoglu and Jacques (2012) conduct a similar analysis in the case of a quasilinear utility function, with a concave benefit function from global abatement and a linear abatement cost function. However, the non-linear case is able to highlight cases where one of the countries is reluctant to sign a specific type of agreement, even if it is better off than in the case of non-cooperation.
} 


\section{The Model}

We consider a transboundary pollution problem across two asymmetric countries $i=1,2$, meaning that the emissions of one country negatively affect the welfare $^{6}$ of the other (existence of externalities). We are interested in a situation where two countries cooperate, taking account of their total welfare in order to mitigate the transboundary pollution problem. The cooperation is realized through international negotiations between the two countries. These countries are supposed to be heterogeneous in terms of their benefits from global abatement, in other words their damage from global pollution, and in terms of their abatement costs.

The utility function of country $i$ can be written as $N B_{i}=B_{i}\left(a_{1}+a_{2}\right)+c_{i}$, where $B_{i}\left(a_{1}+a_{2}\right)$ denotes the benefits from global abatement, $A=a_{1}+a_{2}$, of country $i=1,2$, and $c_{i}$ represents the consumption of a private good. The benefit function from global abatement is assumed to be increasing and concave. In order to take into account the heterogeneity across countries in exposure to global pollution, we write the benefit functions respectively for countries 1 and 2 as follows: $B\left(a_{1}+a_{2}\right)$ and $\alpha B\left(a_{1}+a_{2}\right)$, where $\alpha$ is a positive constant parameter, with $\alpha \leq 1$. This means that country 2 is less sensitive to global pollution than country 1 . In the case of the climate change, this could be explained by a specific geographical location which implies different damage levels.

The abatement cost function is denoted $C_{i}\left(a_{i}\right)$ and is assumed to be increasing and convex. The argument of this function only depends on the individual abatement effort of the country under consideration $a_{i}$. From this perspective, we exclude any possibility of technological transfers or spillovers across the two countries. We assume that, if there is no abatement effort, the abatement cost is null that is $C_{i}(0)=0$, for $i=1,2$. We model the heterogeneity of the abatement costs across countries as: $C\left(a_{1}\right)$ for country 1 and $\delta C\left(a_{2}\right)$ for country 2 , where $\delta$ is a positive constant parameter. We make no assumptions about the value of parameter $\delta$.

First, we examine the threat point of the negotiations if the countries do not cooperate.

\footnotetext{
${ }^{6}$ In our model, individual welfare is the level of utility for each country, which can be written either as the sum of the benefits from global abatement and the consumption of a private good, or the sum of the benefits from global abatement and exogenous initial endowments, minus abatement costs.
} 


\section{$2.1 \quad$ Non-cooperation}

With no cooperation, the objective of each country is to maximize its utility function given the behavior of the other country. We determine the outcome of the Nash equilibrium which represents the threat point of the negotiations. The program of country $i=1,2$ can be written as:

$$
\begin{aligned}
& \quad \underset{a_{i}}{\operatorname{Max}} N B_{i}=\underset{a_{i}}{\operatorname{Max}}\left[B_{i}\left(a_{1}+a_{2}\right)+c_{i}\right] \\
& \Omega_{i} \geq c_{i}+C_{i}\left(a_{i}\right)
\end{aligned}
$$

where $\Omega_{i}$ denotes the exogenous initial endowments of country $i$ used for the consumption of a private good $c_{i}$ and for the abatement $\operatorname{costs} C_{i}\left(a_{i}\right){ }^{7}$

Lemma 1 The abatement levels of countries 1 and 2 are defined respectively by the following system which has a unique solution:

$$
\left\{\begin{aligned}
B^{\prime}(A) & =C^{\prime}\left(a_{1}\right) \\
\alpha B^{\prime}(A) & =\delta C^{\prime}\left(a_{2}\right)
\end{aligned}\right.
$$

Proof: See Appendix A for this proof and that of the uniqueness of the Nash equilibrium.

These conditions give the equality of the individual marginal benefits from global abatement with the individual marginal abatement costs. In fact, when a country abates, it does not take into account the positive effects of its action on the welfare of the other country.

The solution of system 2 gives us respectively abatement for country 1 , and for country 2 and total abatement at the Nash equilibrium, $\hat{a_{1}}, \hat{a_{2}}, \hat{A}$. So we can calculate the welfare level for each country:

$$
\begin{gathered}
\hat{N B_{1}}=B(\hat{A})+\Omega_{1}-C\left(\hat{a_{1}}\right) \\
\hat{N B_{2}}=\alpha B(\hat{A})+\Omega_{2}-\delta C\left(\hat{a_{2}}\right)
\end{gathered}
$$

We now compare the efficiency of the two second-best agreements.

\footnotetext{
${ }^{7} \Omega_{i}$ can be considered an initial endowment of labor, which plays the role of the numeraire. The production of the good is then achieved with a 'one to one' technology (1 unit of input implies 1 unit of production).
} 


\subsection{Cooperation}

It is clear that the agreements with a larger set of negotiation variables will dominate, in terms of total and individual welfare, all other agreements, given the assumptions of concavity of the benefit function and convexity of the cost function. Hence we always obtain superiority, in the Pareto sense, of agreement $\mathrm{D}$ on $\mathrm{U}^{8}$, agreement DT on UT, agreement $\mathrm{UT}$ on $\mathrm{U}$, and agreement DT on D. It thus remains for us to compare, in terms of welfare, the two second-best agreements UT and D. ${ }^{9}$

In the following, we analyze the Nash bargaining solution for the UT agreement.

\subsubsection{Agreement based on a uniform standard with transfers}

We define $\bar{V}=\left[B(2 \bar{a})+c_{1}-\hat{N B_{1}}\right]^{\gamma} \times\left[\alpha B(2 \bar{a})+c_{2}-\hat{N B_{2}}\right]^{1-\gamma}$. In this case, the Nash bargaining solution is written as follows:

$$
\begin{gathered}
\underset{\bar{a}, t}{\operatorname{Max}}[\bar{V}] \\
\text { s.t. } \quad \Omega_{1}=C(\bar{a})+c_{1}+(1+\lambda) t \text { and } \Omega_{2}=\delta C(\bar{a})+c_{2}-t
\end{gathered}
$$

The difference between these budget constraints and those at the threat point is based on the existence of transfer payments $t$. Country 1 gives transfers $(1+\lambda) t$ to country 2 and country 2 receives transfers $t$, where $\lambda \geq 0$ measures the loss in transfer payments. The uniform standard $\bar{a}$ and transfers $t$ are the negotiation variables in this agreement. The first (resp. second) term of $\bar{V}$ represents the gains from cooperation for country 1 (resp. 2). We allow countries to have different bargaining power in the negotiations, namely $\gamma$ for country 1 and $(1-\gamma)$ for country 2 , where $\gamma$ is a positive parameter with

\footnotetext{
${ }^{8}$ Agreement U refers to an agreement based on a uniform standard without transfers, and agreement DT refers to an agreement based on differentiated standards with transfers.

${ }^{9}$ In this paper, we do not try to explain why countries do not agree on the first-best agreement, namely the DT agreement. In a model with informational problems, we could assume that the first-best agreement is costly to implement because of the high negotiation costs associated with multiple negotiation variables. See e.g. Endres (1996) and Harstad (2007).
} 
$0<\gamma<1$. As $\gamma$ increases, the weight of the utility of country 1 increases, and vice versa.

We assume that transfer payments ${ }^{10}$ are imperfect in the sense that country 1 must incur the cost $(1+\lambda) t$ in order to give transfers $t$ to country 2 , with $\lambda \geq 0$. So parameter $\lambda$ measures the imperfection of transfers between the countries. ${ }^{11}$ The extreme case $\lambda=0$ represents the situation with no loss in transfers. The idea of imperfect transfers is similar to the concept of marginal cost of public funds, that is, the social cost of raising one unit of funds. We consider the case where transfers are strictly positive, $t>0^{12}$, that is, where country 1 makes transfer payments to country $2 .{ }^{13}$ This situation corresponds to the case where country 2 has small initial endowments $\left(\Omega_{2}\right.$ small) and/or has less benefit from global abatement $(\alpha<<1)$ than country 1 .

The first-order conditions of this program give us the levels of the uniform standard $\bar{a}$ and transfers $t$ in the agreement based on a uniform standard with transfers. The welfare levels of countries 1 and 2 are then equal to:

$$
\begin{gathered}
N B_{1}^{U}=B(2 \bar{a})+\Omega_{1}-C(\bar{a})-(1+\lambda) t \\
N B_{2}^{U}=\alpha B(2 \bar{a})+\Omega_{2}-\delta C(2 \bar{a})+t
\end{gathered}
$$

\footnotetext{
${ }^{10}$ To introduce monetary transfer payments requires the assumption that the marginal utility for money is constant.

${ }^{11}$ Here, we assume for simplicity that the degree of imperfection of transfers is exogenous. This cost could become endogenous in a political economy model. In that framework, this cost could be related to the rent that the donor country should pay to national lobbies to convince them to make transfer payments to another country. Another possibility would be that the cost is related to the degree of corruption in the recipient country. For a review of the literature on the political economy of IEAs, see Wangler et al. (2011).

${ }^{12}$ This condition is verified for the example presented in Appendix C1.

${ }^{13}$ When transfers are null, the differentiated agreement dominates the uniform agreement in terms of welfare. In that case, the comparison is obvious.
} 
Proposition 1: Existence and Uniqueness of the Bargaining Equilibrium for the UT Agreement

a) The Pareto frontier for the UT agreement is linear

b) The Pareto set for the UT agreement is not empty

c) The bargaining equilibrium for the UT agreement is unique

Proof: Appendix B.

In the following, we analyze the Nash bargaining solution for the D agreement.

\subsubsection{Agreement based on differentiated standards}

We define $V=\left[B\left(a_{1}+a_{2}\right)+c_{1}-\hat{N B_{1}}\right]^{\gamma} \times\left[\alpha B\left(a_{1}+a_{2}\right)+c_{2}-\hat{N B_{2}}\right]^{1-\gamma}$.

Then the Nash bargaining solution can be written as:

$$
\begin{gathered}
\underset{a_{1}, a_{2}}{\operatorname{Max}[V]} \\
\text { s.t. } \quad \Omega_{1}=C\left(a_{1}\right)+c_{1} \text { and } \Omega_{2}=\delta C\left(a_{2}\right)+c_{2}
\end{gathered}
$$

The differentiated standards of the two countries $a_{1}$ and $a_{2}$ are the negotiation variables in this agreement.

The first-order conditions of this program give us the levels of differentiated standards $a_{1}$ and $a_{2}$ in the D agreement. The welfare levels of countries 1 and 2 are then given by:

$$
\begin{gathered}
N B_{1}^{D}=B\left(a_{1}+a_{2}\right)+\Omega_{1}-C\left(a_{1}\right) \\
N B_{2}^{D}=\alpha B\left(a_{1}+a_{2}\right)+\Omega_{2}-\delta C\left(a_{2}\right)
\end{gathered}
$$


Proposition 2: Existence and Uniqueness of the Bargaining Equilibrium for the D Agreement
a) The Pareto frontier for the $D$ agreement is continuous
b) The Pareto set for the $D$ agreement is not empty
c) The Pareto frontier for the $D$ agreement is concave
d) The bargaining equilibrium for the $D$ agreement is unique

Proof: Appendix B.

\section{Comparison of the Total Welfare Levels}

Following Mas-Colell et al. (1995, pp. 825-846), we adopt the term 'social welfare function' to describe the functions of the Nash bargaining solution, $\bar{V}$ and $V$. Here, these functions represent the countries' total welfare.

In this section, we first present the conditions under which the D agreement dominates the UT agreement, in terms of total welfare. To do this, we start by selecting specific values of the variables $a_{1}$ and $a_{2}$, for which the value of the social welfare function $V\left(a_{1}, a_{2}, t=0\right)$ is superior to the maximum value attainable by the social welfare function in the uniform agreement $\bar{V}^{*}\left(\bar{a}^{*}, t^{*}\right)$. Then we can conclude that, under these conditions, the maximum value attainable by the social welfare function in the $\mathrm{D}$ agreement is greater than that attainable in the UT agreement.

We now present the selection of the particular values of the variables $a_{1}$ and $a_{2}$.

Definition $1 \quad$ Let $t^{*}$ and $\bar{a}^{*}$ be the optimal levels of the transfers and the uniform standard in the agreement based on a uniform standard with transfers. We define $a_{1}$ and $a_{2}$ such that:

1) $a_{1}+a_{2}=2 \bar{a}^{*}$

2) $C\left(a_{1}\right)=C\left(\bar{a}^{*}\right)+(1+\lambda) t^{*}$ with $t^{*}>0$.

The particular values of the variables $a_{1}$ and $a_{2}$ are chosen as follows. Given the optimal levels of the transfers and the uniform standard in the UT agreement, $t^{*}$ and $\bar{a}^{*}$, we first construct $a_{1}$ such that $C\left(a_{1}\right)=C\left(\bar{a}^{*}\right)+(1+\lambda) t^{*}$ with $t^{*}>0$. Then, we construct $a_{2}$ in such a way that $\bar{a}^{*}$ is half of the sum of $a_{1}$ and $a_{2}$. 
We assume that $a_{1} \leq 1, a_{2} \underset{*}{\gtrless} 0$ and $t^{*}>0$ (Condition C1). Such conditions imply constraints on $\bar{a}^{*}$ and $t^{*}$. However, it is possible to make assumptions about the fundamental parameters of the model, which are consistent also with our other results. We provide such an example in Appendices $\mathrm{C} 1$ and $\mathrm{C} 2$. For the sake of expositional clarity, we prefer this more general presentation.

It is worth noting that the optimal transfers $t^{*}$ are equal to zero when the countries are symmetric, that is, $\alpha=\delta=1$. In this case, we obtain the equality of the optimal standards in the D and UT agreements, $\bar{a}^{*}=a_{1}^{*}=a_{2}^{*}$, which leads to equivalence of the total welfare levels under these two agreements.

The first condition in Definition 1 implies that the optimal uniform standard is the average of the two particular differentiated standards. The second condition gives us $a_{1}>\bar{a}^{*}$ by the monotonicity of the convex cost function.

We introduce the following principal condition:

$$
\text { Condition C2: } \delta(1+\lambda)\left[C\left(\bar{a}^{*}\right)-C\left(a_{2}\right)\right]>\left[C\left(a_{1}\right)-C\left(\bar{a}^{*}\right)\right]
$$

Proposition 3 provides the sufficient conditions for the superiority of the $\mathrm{D}$ agreement over the UT agreement, in terms of total welfare.

Proposition 3 If Conditions C1 and C2 hold, then the agreement based on differentiated standards without transfers outperforms the agreement based on a uniform standard with transfers, in terms of total welfare.

Proof: Appendix C3.

The central condition of this proposition, Condition C2, means that countries collectively prefer an agreement based on differentiated standards to an agreement based on a uniform standard with transfers, if the cost of transfers weighted with the cost reduction of country 2 associated with adoption of a differentiated standard $\delta\left[C\left(\bar{a}^{*}\right)-C\left(a_{2}\right)\right]$ is higher than the extra cost to country 1 of adopting a differentiated standard $\left(C\left(a_{1}\right)-C\left(\bar{a}^{*}\right)\right)$.

We now provide the conditions under which the UT agreement dominates the $\mathrm{D}$ agreement in terms of total welfare. To do this, we select specific values of the variables $\bar{a}$ and $t$, for which the value of the social welfare function 
$\bar{V}(\bar{a}, t)$ is superior to the maximum value attainable by the social welfare function in the $\mathrm{D}$ agreement $V^{*}\left(a_{1}^{*}, a_{2}^{*}\right)$. Then, we can conclude that, under these conditions, the maximum value attainable by the social welfare function in the UT agreement is greater than that attained in the D agreement. These conditions are symmetric to those presented in the preceding paragraph.

Definition 2 Let $a_{1}^{*}$ and $a_{2}^{*}$ be the optimal levels of standards in the agreement based on differentiated standards without transfers. We define $\bar{a}$ and $t$ such that :

1) $\bar{a}=\frac{a_{1}^{*}+a_{2}^{*}}{2}$

2) $C(\bar{a})+(1+\lambda) t=C\left(a_{1}^{*}\right)$

The particular values of the variables $\bar{a}$ and $t$ are chosen as follows. Given the optimal levels of standards in the $\mathrm{D}$ agreement, $a_{1}^{*}$ and $a_{2}^{*}$, we first construct $\bar{a}$ such that it is half of the sum of $a_{1}^{*}$ and $a_{2}^{*}$. Then, we construct $t$ such that $t=\frac{C\left(a_{1}^{*}\right)-C(\bar{a})}{(1+\lambda)}$.

We assume that $a_{1}^{*}>a_{2}^{*}$ (Condition C3). This condition is verified for the example presented in Appendix C4. Again, for the sake of expositional clarity, we prefer this more general presentation.

We introduce the following principal condition:

$$
\text { Condition C4: } \delta(1+\lambda)\left[C(\bar{a})-C\left(a_{2}^{*}\right)\right]<\left[C\left(a_{1}^{*}\right)-C(\bar{a})\right]
$$

Proposition 4 provides the sufficient conditions for the superiority of the UT agreement over the D agreement, in terms of total welfare.

Proposition 4 If Conditions C3 and $C_{4}$ hold, then the agreement based on a uniform standard with transfers, outperforms the agreement based on differentiated standards without transfers, in terms of total welfare.

Proof: Appendix C5.

The central condition of this proposition, Condition $\mathbf{C 4}$, signifies that the cost of transfers, measured by $\lambda$, must be sufficiently low compared to the ratio for the countries of the difference of the abatement costs between the two agreements. This proposition, which is the alternative to Proposition 3, indicates that, if the cost of transfers is sufficiently low, countries collectively 
prefer an agreement based on a uniform standard with transfers, to the (costeffective) agreement based on differentiated standards. If it is less expensive in terms of transfer costs, to provide incentives for country 2, with weak incentives to abate at a lower cost, then it is in the interest of both countries to sign the uniform agreement. Country 1 pays for relatively inexpensive transfers and benefits from larger total abatement than in the differentiated agreement. Country 2 is compensated by transfer payments for undertaking a higher abatement effort than in the differentiated agreement.

This result can be put in perspective with the findings in the literature on the relative efficiency of second-best agreements. Endres (1997) shows, for quadratic abatement cost and damage cost functions, that the uniform quota agreement (without transfers) could outperform the (cost-efficient) uniform tax agreement, because it yields lower aggregate emissions and a higher level of total welfare. In particular, when the threat point of negotiations is represented by the Nash equilibrium, the uniform quota regime is the only agreement which generates gains from cooperation. Finus and Rundshagen (1998), in a dynamic game with renegotiation-proof equilibria, show that the uniform quota agreement (without transfers) is better in terms of stability, than the uniform tax agreement for global pollution problems with a large number of countries. In the uniform quota agreement, the net benefits of cooperation are more evenly distributed. In this paper, using quite general functional forms, we show that the welfare comparison of the second-best agreements considered is reduced to comparison of the cost of transfers with the relative abatement costs of the countries in the two respective agreements.

We now turn to analysis of the individual welfare levels of the countries under the two second-best agreements.

\section{Comparison of the Individual Welfare Levels}

When the countries sign an agreement, they implicitly agree to a social welfare function that takes the form of a Nash bargaining function. This leads to better outcomes than from the Nash equilibrium in a non-cooperative game. We are interested in situations where both countries are better off signing an agreement, that is, the conditions under which both countries win. However, for a country, the utility obtained in a special agreement could be greater 
than obtained from the other agreement. We conclude this section by focusing on situations where there are conflicts of interest across the countries on the form of the agreement, that is, we investigate the conditions under which one country benefits more by signing one agreement rather than the other agreement. This could explain why some countries are reluctant to sign a particular agreement when they might obtain a higher utility by signing an alternative agreement, even though both countries are better off than in the non-cooperative equilibrium.

More specifically, we are interested in checking the conditions under which both countries can improve their utility by signing the uniform agreement with transfers, compared to their utility under the differentiated agreement. As already mentioned, we consider the case of strictly positive transfers from country 1 to country 2 . As we shall see, this assumption restricts the parameters $\alpha$ and $\Omega_{2}$ to be small enough.

To conduct this analysis, we use the first-order conditions of the two maximization programs (UT and D), and provide a sufficient condition for the efficiency comparison in the Pareto sense. ${ }^{14}$ We establish a result using Conditions (C3) and (C4), thus, assuming the existence of optimal levels of the differentiated standards $a_{1}^{*}$ and $a_{2}^{*}$, and of the optimal levels of the uniform standard $\bar{a}^{*}$ and the transfers $t^{*}$.

\footnotetext{
${ }^{14}$ In the quasi-linear case, this analysis has been conducted using the Pareto frontier (Bayramoglu and Jacques, 2012). In the case of a non-linear cost function, we cannot use the Pareto frontier which becomes non-linear and, thus, difficult to handle analytically. However, interpretation of the results in both the linear and non-linear cases is very similar.
} 
Proposition 5 If conditions $\left(C_{3}\right)$ and $\left(C_{4}\right)$ are verified, then $N B_{2}^{U}$ is greater than $N B_{2}^{D}$. Furthermore, if $\frac{N B_{2}^{D}-N B_{2}}{N B_{2}^{U}-N \hat{B}_{2}}$ is less than $(1+\lambda) \delta \frac{C^{\prime}\left(a_{2}^{*}\right)}{C^{\prime}\left(a_{1}^{*}\right)}$, then $N B_{1}^{U}$ is greater than $N B_{1}^{D}$. Consequently, the agreement based on a uniform standard with transfers Pareto-dominates the agreement based on differentiated standards without transfers.

Proof: Appendix D.

This proposition implies that country 2 prefers the uniform agreement with transfers to the differentiated agreement without transfers under Conditions (C3) and (C4). To obtain a Pareto improvement for country 1 from the UT agreement rather than the D agreement requires an additional condition: $\frac{N B_{2}^{D}-N \hat{B}_{2}}{N B_{2}^{U}-N B_{2}}<(1+\lambda) \delta \frac{C^{\prime}\left(a_{2}^{*}\right)}{C^{\prime}\left(a_{1}^{*}\right)}$. This condition is that the ratio of the gains from cooperation for country 2 in the two agreements must be sufficiently low. In other words, country 2 should improve its utility to a greater extent in the UT agreement compared to the D agreement. Thus, switching from agreement D to agreement UT means the size of the cake (or the total welfare) must increase significantly such that both countries obtain gains from cooperation.

We make two comments on this result:

- the first is related to the reluctance of a country to sign a specific agreement, even if it is better off than in the case of non-cooperation. We know that country 2's utility would improve by signing the UT rather than the D agreement, if Conditions (C3) and (C4) are satisfied. However, if country 1 signs the UT agreement its utility does not necessarily improve compared to signing agreement $\mathrm{D}$. This might induce it not to sign the UT agreement. Using a linear abatement cost function, Bayramoglu and Jacques (2012) show that, like country 2, country 1 is better off by signing the uniform agreement with transfers if Conditions (C3) and (C4) are met.

- the second observation is related to the share of the total surplus between the two countries. According to the first-order conditions of the two programs, given by Equations 24 and 25 (see Appendix D), we have: 


$$
\begin{aligned}
& (\mathrm{D}): \gamma U_{2} \frac{C^{\prime}\left(a_{1}\right)}{\delta C^{\prime}\left(a_{2}\right)}=(1-\gamma) U_{1} \\
& (\mathrm{UT}): \gamma \bar{U}_{2}(1+\lambda)=(1-\gamma) \bar{U}_{1}
\end{aligned}
$$

where $U_{1}=N B_{1}^{D}-N B_{1}$ and $U_{2}=N B_{2}^{D}-N B_{2}$ represent the gains from cooperation of countries 1 and 2 in the $D$ agreement, and $\bar{U}_{1}=N B_{1}^{U T}-N B_{1}$ and $\bar{U}_{2}=N B_{2}^{U T}-N \hat{B}_{2}$ under the UT agreement.

These first-order conditions indicate that if the following condition ${ }^{15}$ holds, $(1+\lambda)<\frac{C^{\prime}\left(a_{1}\right)}{\delta C^{\prime}\left(a_{2}\right)}$, then $\frac{U_{1}}{U_{2}}>\frac{\bar{U}_{1}}{\bar{U}_{2}}$. This means that the relative gains from cooperation of country 1 diminishes from a negotiation on agreement $\mathrm{D}$ to agreement UT. Therefore, the share of the total cake for country 1 reduces with the signature of agreement UT under the above condition. However, this cannot be considered an obstacle to participation in agreement UT for country 1 because, in absolute terms, this country could be better off in the UT agreement than in the D agreement.

\section{A Numerical Example}

Our aim is to compare the outcomes of the UT and D agreements using a simple numerical example. We take the case of a linear benefit function from global abatement and a quadratic abatement cost function. The utility function of the countries 1 and 2 can be written as follows:

$$
\begin{aligned}
& N B_{1}=e\left(a_{1}+a_{2}\right)-\frac{c}{2} a_{1}^{2} \\
& N B_{2}=\alpha e\left(a_{1}+a_{2}\right)-\frac{\delta c}{2} a_{2}^{2}
\end{aligned}
$$

For simplicity, we consider that the countries have identical negotiation power, that is, $\gamma=1 / 2$. The threat point of negotiation is assumed to be

\footnotetext{
${ }^{15}$ If Conditions C3 and C4 are satisfied, this condition always holds (see the proof of Proposition 5 in Appendix D).
} 
the situation where neither country abates, and hence has zero utility at the Nash equilibrium, that is, $\hat{a}_{1}=\hat{a}_{2}=\hat{N B_{1}}=\hat{N B_{2}}=0$. We search for parameter constellations which respect a number of constraints for the two cooperative equilibria: quasi-concavity of the program, positive abatement standards and utility levels, positivity of transfers in the UT agreement, and $a_{1}^{*}>a_{2}^{*}$ (Condition C3) in agreement D. Example 1 (resp. 2) illustrates the case where total welfare is higher (resp. lower) under the UT agreement than under the D agreement.

Example 1: $\quad \lambda=0.1, c=0.1, e=1, \alpha=0.1, \delta=0.3$, we obtain: $a_{1}=16, a_{2}=8, A^{D}=a_{1}+a_{2}=24, N B_{1}^{D}=10.66, N B_{2}^{D}=1.52, V=16.20$; $\bar{a}=16, A^{U}=2 \bar{a}=32, t=9, N B_{1}^{U}=9.26, N B_{2}^{U}=8.42, \bar{V}=77.97$.

Example 2: $\quad \lambda=5, c=0.1, e=1, \alpha=0.1, \delta=0.3$, we obtain: $a_{1}=16.99, a_{2}=8.1, A^{D}=a_{1}+a_{2}=25.09, N B_{1}^{D}=10.66, N B_{2}^{D}=1.52, V=$ $16.2 ; \bar{a}=11, A^{U}=2 \bar{a}=22, t=1.2, N B_{1}^{U}=9.14, N B_{2}^{U}=1.52, \bar{V}=13.93$.

With the exception of parameter $\lambda$, the values of all the parameters are the same in the two examples. In Example 2, the parameter of cost of transfers $(\lambda)$ is higher than in Example 1.

We checked that Condition C4 is satisfied in Example 1, but is violated in Example 2. Hence, these numerical examples illustrate the theoretical result of Proposition 4 that, when the cost of transfers is sufficiently low, countries collectively prefer an agreement based on a uniform standard with transfers, to an agreement based on differentiated standards. In Example 1, country 2 is better off in agreement UT than in agreement D, while country 1 has higher utility in agreement $\mathrm{D}$. In this case, country 1 might be reluctant to sign agreement UT. This finding illustrates the theoretical result of Proposition 5 , because the additional condition, $\frac{N B_{2}^{D}}{N B_{2}^{U}}<(1+\lambda) \delta \frac{a_{2}^{*}}{a_{1}^{*}}$, to obtain a Pareto improvement for country 1 from the UT agreement rather than the D agreement, is not fulfilled in this case. In Example 2, country 2 is indifferent between the two agreements, but country 1 prefers agreement D.

These examples also allow us to compare the total mitigation achieved by the two agreements. In Example 1, agreement UT yields larger total abatement than agreement D. In Example 2, the reverse situation applies. In Example 1, the low level of transfer costs allows country 1 to make significant transfer payments to country 2. These payments give the appropriate 
incentives for country 2 to abate a significant amount of pollution at lower cost. Under the parameter constellations considered in Example 1, agreement UT leads to a better environmental quality and a higher level of total welfare.

\section{Conclusion}

In this paper, we compared the relative efficiency of two second-best agreements frequently observed in reality, for two asymmetric countries faced with a transboundary pollution problem. The two agreements studied include one based on a uniform standard with monetary transfers and one based on differentiated standards without transfers. We paid particular attention to the role of the costs associated with transfer payments across countries in the choice between these agreements. The analysis was based on a negotiation game and the generalized Nash bargaining solution (Nash, 1953) as the equilibrium. The analysis was carried out for a concave benefit function from global abatement and a convex abatement cost function.

The findings from our analysis of total welfare show that, if the cost of transfers is sufficiently low, countries collectively prefer the agreement based on a uniform standard with transfers to the agreement based on differentiated standards. More specifically, the cost of transfers must be sufficiently low compared to the ratio for the countries of the difference of the abatement costs between the two agreements.

Analysis of individual welfare shows that the same condition allows the 'less environmentally conscious country' ${ }^{\prime}$ to improve its welfare under the differentiated agreement compared to the uniform agreement. An additional condition is needed for the 'environmentally conscious country' to obtain a welfare improvement under the uniform agreement compared to the differentiated agreement. This condition is that total welfare (or the total cake) must increase significantly in the agreement based on a uniform standard with transfers. Overall, monetary transfers which are less costly for the donor country, contribute to compensating the additional abatement cost of the recipient country in the uniform agreement, because, in this case, the level of abatement of the recipient country is higher than that in the differentiated agreement. These results indicate first that asymmetric countries with different abatement technology and pollution damage, might be attracted to

\footnotetext{
${ }^{16}$ This terminology is taken from Petrakis and Xepapadeas (1996).
} 
signing an agreement based on a uniform standard. This holds if the uniform agreement includes a side payment scheme across countries. In addition, transfer payments should not be very costly - in terms of administrative or political costs - to implement.

It seems that the costs of transfer payments across countries will play a crucial role in the success of future climate change negotiations. The current financing of abatement expenditures appears complex given the high number of bilateral agreements across countries. The climate negotiations held in Cancun in 2010 established a Green Climate Fund (GCF) to finance developing countries' adaptation investments related to climate change. It is hoped that this will reduce the cost of the side payments included in the provisions of several bilateral agreements. In particular, 'the formation of the new fund should serve to simplify the intricate network of funding mechanisms and bilateral agreements that currently provide low carbon and climate adaptation investment for developing countries' (Businessgreen, 2010). The climate negotiations held in Warsaw in 2013 agreed a text calling for 'ambitious and timely contributions by developed countries to ensure the effective operationalization of the fund and the mobilization of initial resources before the next round of talks in Peru' (Climate Policy Watcher, 2013 ${ }^{17}$ ). The motivation for this new fund is the Multilateral Fund of the Montreal Protocol (1987). The Multilateral Fund allowed the financing of the additional costs induced by the implementation of the Protocol in developing countries. It has contributed to the participation of these countries in the Protocol, and the implementation of investment projects in abatement (Luken and Grof, 2006).

\footnotetext{
${ }^{17}$ http://www.climate-policy-watcher.org/?q=node/584.
} 


\section{References}

[1] Barrett, S. (1994) Self-enforcing international environmental agreements, Oxford Economic Papers 46, 878-894.

[2] Barrett, S. (2001) International cooperation for sale, European Economic Review 45, 1835-1850.

[3] Bayramoglu, B., Jacques, J-F. (2011) The role of fixed cost in international environmental negotiations Environment and Development Economics 16(2), 221-238.

[4] Bayramoglu, B., Jacques, J-F. (2012) Les négociations internationales sur l'environnement: norme uniforme et normes différenciées, Revue d'Economie Politique 122(6), 943-969.

[5] Brandt, U-S. (2004) Are uniform solutions focal? - The case of international environmental agreements, Environmental and Resource Economics 25, 357-376.

[6] Buchholz, W., Haupt, A., Peters, W. (2005) International environmental agreements and strategic voting, Scandinavian Journal of Economics 107(1), 175-195.

[7] Businessgreen (2010) Cancun Green Fund to dominate global climate finance, James Murray, 13 December 2010.

[8] Caparros, A., Péreau, J-C., Tazdait, T. (2004) North-South climate change negotiations: A sequential game with asymmetric information, Public Choice 121, 455-480.

[9] Carraro, C., Siniscalco, D. (1993) Strategies for the international protection of the environment, Journal of Public Economics 52, 309-328.

[10] Chander, P., Tulkens, H. (1995) A core-theoretic solution for the design of cooperative agreements on transfrontier pollution, International Tax and Public Finance 2, 279-294.

[11] Chander, P., Tulkens, H. (1997) The core of an economy with multilateral environmental externalities, International Journal of Game Theory 26, 379-401. 
[12] Chen, Z. (1997) "Negotiating an agreement on global warming: a theoretical analysis" Journal of Environmental Economics and Management 32, 170-188.

[13] Compte, O., Jéhiel, P. (1997) International negotiations and dispute resolution mechanisms: the case of environmental negotiations. In: C. Carraro (ed) International Environmental Negotiations: Strategic Policy Issues, Cheltenham, Edward Elgar.

[14] Copeland, B., Taylor, S. (2005) Free trade and global warming: a trade theory view of the Kyoto protocol, Journal of Environmental Economics and Management 49, 205-234.

[15] Courtois, P., Tazdait, T. (2011) Bargaining over a climate deal: deadline and delay, Annals of Operations Research.

[16] Dannenberg, A., Lange, A., Sturm, B. (2010) On the formation of coalitions to provide public goods - experimental evidence from the lab, NBER Working Paper Series, Working Paper 15967.

[17] Endres, A. (1996) Designing a greenhouse treaty: some economic problems. In: E.Eide and R. van der Bregh (eds) Law and Economics of the Environment, Oslo: Jurudisk Forlag, pp 201-224.

[18] Endres, A. (1997) Negotiating a climate convention: The role of prices and quantities, International Review of Law and Economics 17,147-156.

[19] Eyckmans, J. (1999) Strategy proof uniform effort sharing schemes for transfrontier pollution problems, Environmental and Resource Economics 14, 165-189.

[20] Finus, M., Rundshagen, B. (1998) Toward a positive theory of coalition formation and endogenous instrumental choice in global pollution control, Public Choice 96, 145-186.

[21] Finus, M. (2001), Game Theory and International Environmental Cooperation, Cheltenham: Edwar Elgar, Inc.

[22] Finus, M. (2008), Game theoretic research on the design of international environmental agreements: insights, critical remarks, and future challenges, International Review of Environmental and Resource Economics 2, 29-67. 
[23] Fuentes-Albero, C., Rubio, S. (2010) Can international environmental cooperation be bought?, European Journal of Operational Research 202, 255-264.

[24] Harstad, B. (2007) Harmonization and side payments in political cooperation, American Economic Review 97, 871-889.

[25] Hoel, M. (1991) Global environmental problems: the effects of unilateral actions taken by one country, Journal of Environmental Economics and Management 20, 55-70.

[26] Hoel, M. (1992) International environment conventions: the case of uniform reductions of emissions, Environmental and Resource Economics 2, 141-159.

[27] Jéhiel, P. (1997) Bargaining between benevolent jurisdictions or when delegation induces inefficiencies, Journal of Public Economics 65, 61-74.

[28] Larson, B.A., Tobey, J.A. (1994) Uncertain climate change and the international policy response, Ecological Economics 11, 77-84.

[29] Luken, R., Grof, T. (2006) The Montreal Protocol's multilateral fund and sustainable development, Ecological Economics, vol.56, issue 2, 241255.

[30] Maler, K-G. (1989) The acid rain game. In: H. Folmer and E. Van Ierland (eds), Valuation Methods and Policy Making in Environmental Economics, Amsterdam, Elsevier, pp 231-251.

[31] Mas-Colell, A., Whinston, M.D., Green, J.R. (1995) Microeconomic Theory. Oxford University Press, New York.

[32] Nash, J.F. (1953) Two-person cooperative games, Econometrica 21, 128-140.

[33] Newbery, D-M. (1990) Acid rain, Economic Policy 5, 297-346.

[34] Petrakis, E., Xepapadeas, A. (1996) Environmental consciousness and moral hazard in international agreements to protect the environment, Journal of Public Economics 60, 95-110. 
[35] Wangler, L., Altamirano-Cabrera, J-C., Weikard, H-P. (2011) The political economy of international environmental agreements: a survey, JENA Economic Research Papers 2011-038, Jena, Germany.

[36] Weikard, H. P. (2009) Cartel stability under optimal sharing rule, Manchester School 77, 575-593. 


\section{APPENDIX}

\section{A- Nash Equilibrium \\ Proof of Lemma 1}

The objective of country 1 is to maximize its utility function with respect to its budget constraint, taking as given the level of abatement of country 2, $a_{2}: \operatorname{Max}_{a_{1}}\left[B\left(a_{1}+a_{2}\right)+\Omega_{1}-C\left(a_{1}\right)\right]$.

The first-order condition of this program is the following: $B^{\prime}(A)=C^{\prime}\left(a_{1}\right)$. Similarly, the first-order condition of the program for country 2 is the following: $\alpha B^{\prime}(A)=\delta C^{\prime}\left(a_{2}\right)$.

Uniqueness of the Nash equilibrium

The equilibrium is defined by:

$$
\begin{aligned}
B^{\prime}\left(a_{1}+a_{2}\right) & =C^{\prime}\left(a_{1}\right)(1) \\
\alpha B^{\prime}\left(a_{1}+a_{2}\right) & =\delta C^{\prime}\left(a_{2}\right)(2)
\end{aligned}
$$

Suppose there is another equilibrium:

$$
\begin{aligned}
B^{\prime}\left(\bar{a}_{1}+\bar{a}_{2}\right) & =C^{\prime}\left(\bar{a}_{1}\right)(3) \\
\alpha B^{\prime}\left(\bar{a}_{1}+\bar{a}_{2}\right) & =\delta C^{\prime}\left(\bar{a}_{2}\right)(4)
\end{aligned}
$$

Assume that $a_{1}>\bar{a}_{1}$, so $B^{\prime}\left(a_{1}+a_{2}\right)>B^{\prime}\left(\bar{a}_{1}+\bar{a}_{2}\right)$ because of (1) and (3), and the convexity of $C($.$) . Consequently, a_{2}>\bar{a}_{2}$ because of (2) and (4), and the convexity of $C($.$) . So a_{1}+a_{2}>\bar{a}_{1}+\bar{a}_{2}$, then $B^{\prime}\left(a_{1}+a_{2}\right)<B^{\prime}\left(\bar{a}_{1}+\bar{a}_{2}\right)$ because of the concavity of $B($.$) , which is contradictory to a_{1}>\bar{a}_{1}$ and $B^{\prime}\left(a_{1}+a_{2}\right)>B^{\prime}\left(\bar{a}_{1}+\bar{a}_{2}\right)$.

Assume that $a_{1}<\bar{a}_{1}$, so $B^{\prime}\left(a_{1}+a_{2}\right)<B^{\prime}\left(\bar{a}_{1}+\bar{a}_{2}\right)$ because of (1) and (3), and the convexity of $C($.$) . Consequently, a_{2}<\bar{a}_{2}$ because of (2) and (4), and the convexity of $C($.$) . So a_{1}+a_{2}<\bar{a}_{1}+\bar{a}_{2}$, then $B^{\prime}\left(a_{1}+a_{2}\right)>B^{\prime}\left(\bar{a}_{1}+\bar{a}_{2}\right)$ because of the concavity of $B($.$) , which is contradictory to a_{1}<\bar{a}_{1}$ and $B^{\prime}\left(a_{1}+a_{2}\right)<B^{\prime}\left(\bar{a}_{1}+\bar{a}_{2}\right)$.

This proof shows that the Nash equilibrium is unique under the standard assumptions of a concave benefit function and a convex cost function.

B- Existence and Uniqueness of the Cooperative Equilibria 


\section{Proof of Proposition 1a}

The Pareto optimality results from the following program

$$
\begin{aligned}
\max _{\bar{a}, t} N B_{1}=\max _{\bar{a}, t}\left[B(2 \bar{a})+c_{1}\right] \\
\text { s.t. } \quad\left\{\begin{array}{c}
\Omega_{1}=c_{1}+C(\bar{a})+(1+\lambda) t \\
\Omega_{2}=c_{2}+\delta C(\bar{a})-t \\
N B_{2}=\alpha B(2 \bar{a})+c_{2} \geq N B_{2}
\end{array}\right.
\end{aligned}
$$

where $\overline{\overline{N B}}$ is exogenous.

If we use the first two constraints, the program becomes

$$
\begin{aligned}
& \max _{\bar{a}, t} N B_{1}=\max _{\bar{a}, t}\left[B(2 \bar{a})+\Omega_{1}-C(\bar{a})-(1+\lambda) t\right] \\
& \text { s.t. } \quad N B_{2}=\alpha B(2 \bar{a})+\Omega_{2}-\delta C(\bar{a})+t \geq N \overline{\overline{B_{B}}}
\end{aligned}
$$

The Lagrangian of this maximization problem is: $L=\left[B(2 \bar{a})+\Omega_{1}-\right.$ $C(\bar{a})-(1+\lambda) t]+\rho\left[\alpha B(2 \bar{a})+\Omega_{2}-\delta C(\bar{a})+t-\overline{\bar{E}} B_{2}\right]$, where $\rho$ is the multiplier associated with the constraint.

The first-order conditions (FOCs) with respect to $\bar{a}$ and $t$ give:

$$
2(\alpha(1+\lambda)+1) B^{\prime}(2 \bar{a})=(1+\delta(1+\lambda)) C^{\prime}(\bar{a})
$$

The total differential of $N B_{1}$ is equal to: $d\left(N B_{1}\right)=\left[2 B^{\prime}(2 \bar{a})-C^{\prime}(\bar{a})\right] d \bar{a}-$ $(1+\lambda) d t$, and that of $N B_{2}$ is equal to: $d\left(N B_{2}\right)=\left[2 \alpha B^{\prime}(2 \bar{a})-\delta C^{\prime}(\bar{a})\right] d \bar{a}+d t$.

On the Pareto frontier, we have

$$
\begin{aligned}
& d\left(N B_{1}\right)=\frac{C^{\prime}(\bar{a})(1+\lambda)(\delta-\alpha)}{(\alpha(1+\lambda)+1)} d \bar{a}-(1+\lambda) d t \\
& d\left(N B_{2}\right)=\frac{C^{\prime}(\bar{a})(\alpha-\delta)}{(\alpha(1+\lambda)+1)} d \bar{a}+d t
\end{aligned}
$$

So along the Pareto frontier $\frac{d N B_{1}}{d N B_{2}}=-(1+\lambda)$. Hence, the Pareto frontier is a decreasing line. 


\section{Proof of Proposition 1b}

We consider two points on the Pareto frontier. Consider the case where $\delta C(\bar{a})=\Omega_{2}$ when the private consumption of country 2 and the transfers are both equal to 0 . So the point $\left(N B_{1, \max }, N B_{2, \min }\right)$ defined by $N B_{1, \max }=$ $B(2 \bar{a})+\Omega_{1}-C(\bar{a})$ and $N B_{2}=\alpha B(2 \bar{a})$ with $\bar{a}$ defined by $\delta C(\bar{a})=\Omega_{2}$ is on the Pareto frontier. The point $\left(N B_{1, \min }, N B_{2, \max }\right)$ defined by $N B_{1, \min }=B(2 \bar{a})$ and $N B_{2, \max }=\alpha B(2 \bar{a})+\Omega_{2}-\delta C(\bar{a})+t$ with $\bar{a}$ defined by $2 \alpha B^{\prime}(2 \bar{a})=\delta C^{\prime}(\bar{a})$ and $t$ defined by $(1+\lambda) t+C(\bar{a})=\Omega_{1}$ is also on the Pareto frontier. These two points are different so the Pareto set is not empty.

\section{Proof of Proposition 1c}

The social welfare function is strictly concave and the Pareto set is convex. So there exists a unique bargaining equilibrium for agreement UT.

\section{Proof of Proposition 2a}

The Pareto optimality results from the following program

$$
\begin{gathered}
\max _{a_{1}, a_{2}} N B_{1}=\max _{a_{1}, a_{2}}\left[B\left(a_{1}+a_{2}\right)+c_{1}\right] \\
\text { s.t. }\left\{\begin{array}{c}
\Omega_{1}=c_{1}+C\left(a_{1}\right) \\
\Omega_{2}=c_{2}+\delta C\left(a_{2}\right) \\
N B_{2}=\alpha B\left(a_{1}+a_{2}\right)+c_{2} \geq N B_{2}
\end{array}\right.
\end{gathered}
$$

where $\overline{\overline{N B}}$ is exogenous.

If we use the first two constraints, the program becomes

$$
\begin{array}{ll}
\max _{a_{1}, a_{2}} & N B_{1}=\max _{a_{1}, a_{2}}\left[B\left(a_{1}+a_{2}\right)+\Omega_{1}-C\left(a_{1}\right)\right] \\
\text { s.t. } & N B_{2}=\alpha B\left(a_{1}+a_{2}\right)+\Omega_{2}-\delta C\left(a_{2}\right) \geq N \overline{\bar{B}}_{2}
\end{array}
$$

The Lagrangian of this maximization problem is: $L=\left[B\left(a_{1}+a_{2}\right)+\Omega_{1}-\right.$ $\left.C\left(a_{1}\right)\right]+\rho\left[\alpha B\left(a_{1}+a_{2}\right)+\Omega_{2}-\delta C\left(a_{2}\right)-\overline{\bar{N}} B_{2}\right]$, where $\rho$ is the multiplier associated with the constraint.

The first-order conditions (FOCs) with respect to $a_{1}$ and $a_{2}$ are respectively: $B^{\prime}\left(a_{1}+a_{2}\right)-C^{\prime}\left(a_{1}\right)=-\rho \alpha B^{\prime}\left(a_{1}+a_{2}\right)$, and $B^{\prime}\left(a_{1}+a_{2}\right)=-\rho\left[\alpha B^{\prime}\left(a_{1}+\right.\right.$ $\left.\left.a_{2}\right)-\delta C^{\prime}\left(a_{2}\right)\right]$. 
The ratio of these FOCs gives

$$
\frac{1}{B^{\prime}\left(a_{1}+a_{2}\right)}=\frac{\alpha}{\delta C^{\prime}\left(a_{2}\right)}+\frac{1}{C^{\prime}\left(a_{1}\right)}
$$

The total differential of $N B_{1}$ is equal to: $d N B_{1}=B^{\prime}\left(a_{1}+a_{2}\right)\left(d a_{1}+d a_{2}\right)-$ $C^{\prime}\left(a_{1}\right) d a_{1}$, and that of $N B 2$ is equal to: $d N B_{2}=\alpha B^{\prime}\left(a_{1}+a_{2}\right)\left(d a_{1}+d a_{2}\right)-$ $\delta C^{\prime}\left(a_{2}\right) d a_{2}$.

On the Pareto frontier, we have

$$
\begin{gathered}
d\left(N B_{1}\right)=\frac{\delta C^{\prime}\left(a_{1}\right) C^{\prime}\left(a_{2}\right)}{\delta C^{\prime}\left(a_{2}\right)+\alpha C^{\prime}\left(a_{1}\right)}\left(d a_{1}+d a_{2}\right)-C^{\prime}\left(a_{1}\right) d a_{1} \\
d\left(N B_{2}\right)=\frac{\alpha \delta C^{\prime}\left(a_{1}\right) C^{\prime}\left(a_{2}\right)}{\delta C^{\prime}\left(a_{2}\right)+\alpha C^{\prime}\left(a_{1}\right)}\left(d a_{1}+d a_{2}\right)-\delta C^{\prime}\left(a_{2}\right) d a_{2} \\
d\left(N B_{1}\right)=\frac{\delta C^{\prime}\left(a_{1}\right) C^{\prime}\left(a_{2}\right) d a_{2}-\alpha C^{\prime}\left(a_{1}\right) C^{\prime}\left(a_{1}\right) d a_{1}}{\delta C^{\prime}\left(a_{2}\right)+\alpha C^{\prime}\left(a_{1}\right)} \\
d\left(N B_{2}\right)=\frac{\alpha \delta C^{\prime}\left(a_{1}\right) C^{\prime}\left(a_{2}\right) d a_{1}-\delta C^{\prime}\left(a_{2}\right) \delta C^{\prime}\left(a_{2}\right) d a_{2}}{\delta C^{\prime}\left(a_{2}\right)+\alpha C^{\prime}\left(a_{1}\right)}
\end{gathered}
$$

Along the Pareto frontier, we have $\frac{d N B_{1}}{d N B_{2}}=-\frac{1}{\delta} \frac{C^{\prime}\left(a_{1}\right)}{C^{\prime}\left(a_{2}\right)}$. The Pareto frontier is differentiable, thus it is continuous.

\section{Proof of Proposition 2b}

We consider two points on this frontier : for $N B_{2}$, consider the case where $\delta C\left(a_{2}\right)=\Omega_{2}$ when the private consumption is equal to 0 . At that point, the maximum of $N B_{1}$ is attainable for $B^{\prime}\left(a_{1}+a_{2}\right)=C^{\prime}\left(a_{1}\right)$. So the point $\left(N B_{1, \max }, N B_{2, \min }\right)$ defined by $N B_{1, \max }=B\left(a_{1}+a_{2}\right)+\Omega_{1}-C\left(a_{1}\right)$ and $N B_{2, \min }=\alpha B\left(a_{1}+a_{2}\right)$ with $a_{2}$ defined by $\delta C\left(a_{2}\right)=\Omega_{2}$ and $a_{1}$ defined by $B^{\prime}\left(a_{1}+a_{2}\right)=C^{\prime}\left(a_{1}\right)$, is on the Pareto frontier. Similarly, the point $\left(N B_{1, \min }, N B_{2, \max }\right)$ defined by $N B_{1, \min }=B\left(a+a_{2}\right)$ and $N B_{2, \max }=\alpha B\left(a_{1}+\right.$ $\left.a_{2}\right)+\Omega_{2}-\delta C\left(a_{2}\right)$ with $a_{1}$ defined by $C\left(a_{1}\right)=\Omega_{1}$ and $a_{2}$ defined by $\alpha B^{\prime}\left(a_{1}+\right.$ $\left.a_{2}\right)=\delta C^{\prime}\left(a_{2}\right)$ is on the Pareto frontier. So the Pareto set is not empty because these two points are different.

\section{Proof of Proposition 2c}

Assume that there exists an interval $\left[\underline{N B}_{2}, \overline{N B_{2}}\right]$ on which the Pareto frontier is convex. Consider a point on this interval $\widetilde{N B}_{2}$ which is a convex combination of $\underline{N B_{2}}$ and $\overline{N B_{2}}$, that is, $\widetilde{N B}_{2}=\gamma \underline{N B_{2}}+(1-\gamma) \overline{N B_{2}}$. 
By definition of the local convexity of the Pareto frontier, the same convex combination of $N B_{1}$, denoted $\widehat{N B_{1}}=\gamma N B_{1}+(1-\gamma) \overline{N B_{1}}$, is greater than the corresponding point $\widetilde{\widetilde{N B_{1}}}$ of the Pareto frontier defined by

$$
\begin{aligned}
\widetilde{\widetilde{N B_{1}}} & =\operatorname{Max} N B_{1} \\
\text { s.t. } N B_{2} & \geq \widetilde{N B}_{2}
\end{aligned}
$$

Moreover the concavity of the function $N B_{1}=B\left(a_{1}+a_{2}\right)-C\left(a_{1}\right)+\Omega_{1}$ implies that $\widetilde{N B}_{1}=\gamma \underline{N B_{1}}+(1-\gamma) \overline{N B_{1}}<N B_{1}\left(\gamma \underline{a_{1}}+(1-\gamma) \overline{a_{1}}, \gamma \underline{a_{2}}+(1-\right.$ $\left.\gamma) \overline{a_{2}}\right)=\overline{\overline{N B_{1}}}$. This level of utility is attainable because $\overline{\overline{a_{1}}}=\gamma \underline{a_{1}}+(1-\gamma) \overline{a_{1}}$ and $\overline{\overline{a_{2}}}=\gamma \underline{a_{2}}+(1-\gamma) \overline{a_{2}}$ are convex combinations of possible abatements in the budget set. Moreover $\overline{\overline{N B_{2}}}\left(\gamma \underline{a_{1}}+(1-\gamma) \overline{a_{1}}, \gamma \underline{a_{2}}+(1-\gamma) \overline{a_{2}}\right) \geq \widetilde{N B_{2}}$ by the concavity of the function $N \overline{B_{2}}$. So for the abatements $\overline{\overline{a_{1}}}$ and $\overline{\overline{a_{2}}}$ we have $\overline{\overline{N B_{2}}} \geq \widetilde{N B_{2}}$ and $\widetilde{\widetilde{N B_{1}}}<\widetilde{N B_{1}}<\overline{\overline{N B_{1}}}$ which is contradictory with the construction of the Pareto frontier given by program 13, because we have found a level of utility $\overline{\overline{N B_{1}}}$ associated with a level $\overline{\overline{N B_{2}}}$ which is above the Pareto frontier. So the Pareto frontier cannot be convex locally. It is concave and the Pareto set is convex.

\section{Proof of Proposition 2d}

The social welfare function is strictly concave and the Pareto set is convex. So there exists a unique bargaining equilibrium for agreement D.

\section{C- Total Welfare}

The goal of Appendix $\mathrm{C}$ is to exhibit simple examples which fulfill Conditions $\mathrm{C} 1$ and $\mathrm{C} 3$.

C1- Positivity of transfers in the UT agreement: the case with a linear cost, and $\alpha \simeq 0, \delta<1, \gamma=1 / 2$ (Condition C1)

Here, we assume a linear cost function $C(a)=c a$, where $c$ is a positive parameter. We show that when the benefits from global abatement of country 2 are low $(\alpha \simeq 0)$ and the abatement costs of country 2 are lower than that of country $1(\delta<1)$, then the transfers from country 1 to country 2 are positive.

For the uniform agreement with transfers from country 1 to country 2 , the Nash bargaining problem with equal bargaining powers $(\gamma=1 / 2)$ leads to the following first-order condition with respect to transfer $(t)$ : 


$$
\begin{aligned}
& \frac{\partial \bar{V}}{\partial t}=0 \Longleftrightarrow-(1+\lambda)\left[\alpha B(2 \bar{a})+\Omega_{2}-\delta c \bar{a}+t-N B_{2}\right]+ \\
& {\left[B(2 \bar{a})+\Omega_{1}-c \bar{a}-(1+\lambda) t-N B_{1}\right]=0} \\
& \Longleftrightarrow 2(1+\lambda) t=B(2 \bar{a})(1-(1+\lambda) \alpha)-(1+\lambda) \Omega_{2}+\Omega_{1}+c \bar{a}((1+\lambda) \delta-1) \\
& +\left((1+\lambda) N B_{2}-N B_{1}\right)
\end{aligned}
$$

Concerning the term $\left((1+\lambda) \hat{N B_{2}}-N B_{1}\right)$, the welfare levels at the threat point are given by: $\hat{N B_{1}}=B\left(B^{\prime-1}(c)\right)+\Omega_{1}-c B^{\prime-1}(c)$ and $\hat{N B_{2}}=$ $\alpha B\left(B^{\prime-1}(c)\right)+\Omega_{2}$. That gives us:

$$
\begin{gathered}
\left((1+\lambda) \hat{N B_{2}}-\hat{N} B_{1}\right)=(1+\lambda)\left(\alpha B\left(B^{\prime-1}(c)\right)+\Omega_{2}\right)-B\left(B^{\prime-1}(c)\right)-\Omega_{1}+c B^{\prime-1}(c) \\
\Longleftrightarrow(1+\lambda) \Omega_{2}-\Omega_{1}+(1+\lambda) \alpha B\left(B^{\prime-1}(c)-B\left(B^{\prime-1}(c)\right)+c B^{\prime-1}(c)\right.
\end{gathered}
$$

We make the following simplifying assumption: $\alpha \simeq 0$. Then, the level of transfers becomes: $2(1+\lambda) t \simeq B(2 \bar{a})-c \bar{a}(1-(1+\lambda) \delta)-B\left(B^{\prime-1}(c)\right)+c B^{\prime-1}(c)$.

The expression $[B(2 \bar{a})-c \bar{a}(1-(1+\lambda) \delta)]$ is superior or equal to $\left(N B_{1}-\right.$ $\left.\Omega_{1}\right)$ because $B(2 \bar{a})-c \bar{a}(1-(1+\lambda) \delta) \geq B(2 \bar{a})-c \bar{a}-(1+\lambda) t$. Furthermore, the expression $\left[-B\left(B^{\prime-1}(c)\right)+c B^{\prime-1}(c)\right]$ is equal to $\left(-N B_{1}+\Omega_{1}\right)$. We thus have,

$$
2(1+\lambda) t \simeq B(2 \bar{a})-c \bar{a}(1-(1+\lambda) \delta)-B\left(B^{\prime-1}(c)\right)+c B^{\prime-1}(c) \geq N B_{1}-N \hat{B}_{1}
$$

When country 1 signs a UT agreement, its utility is greater than that at the threat point, hence the transfers from country 1 to country 2 are positive.

C2- The conditions $a_{1} \leq 1$ and $a_{2} \geq 0$ (Condition C1)

In order to have $a_{1} \leq 1$ and $a_{2} \geq 0$, it is sufficient, for instance, that in the case with a linear cost function, we have $\alpha \simeq 0 ;(1+\lambda) \delta \simeq 1,(1+\lambda) \delta$ 
could be less than or greater than 1 , in the neighborhood of 1 ; $c$ must be sufficiently large and the function $B^{\prime-1}($.$) must be sufficiently small.$

The second condition of Definition 1 gives us:

$C\left(a_{1}\right)=C\left(\bar{a}^{*}\right)+(1+\lambda) t^{*} \Longleftrightarrow c a_{1}=c \bar{a}^{*}+(1+\lambda) t^{*} \Longleftrightarrow a_{1}=\bar{a}^{*}+\frac{(1+\lambda) t^{*}}{c}$

Similarly, the first condition of Definition 1 gives:

$$
a_{2}=2 \bar{a}^{*}-a_{1} \Longleftrightarrow a_{2}=2 \bar{a}^{*}-\bar{a}^{*}-\frac{(1+\lambda) t^{*}}{c} \Longleftrightarrow a_{2}=\bar{a}^{*}-\frac{(1+\lambda) t^{*}}{c}
$$

One knows, from proof $\mathrm{C}$, that the first-order conditions related to the Nash bargaining solution with equal bargaining powers $(\gamma=1 / 2)$ in the UT agreement imply:

$$
\begin{gathered}
\left(2 B^{\prime}(2 \bar{a})-c\right)\left[\alpha B(2 \bar{a})+\Omega_{2}-\delta c \bar{a}+t-N B_{2}\right]+ \\
\left(2 \alpha B^{\prime}(2 \bar{a})-\delta c\right)(1+\lambda)\left[\alpha B(2 \bar{a})+\Omega_{2}-\delta c \bar{a}+t-N B_{2}\right]=0 \\
\Longleftrightarrow\left(2 B^{\prime}(2 \bar{a})-c\right)+\left(2 \alpha B^{\prime}(2 \bar{a})-\delta c\right)(1+\lambda)=0 \Longleftrightarrow B^{\prime}(2 \bar{a})=\frac{c(1+\delta(1+\lambda))}{2(1+\alpha(1+\lambda))}
\end{gathered}
$$

We make the following assumptions: $\delta(1+\lambda) \simeq 1$ and $\alpha \simeq 0$. Under these assumptions, the expression of the marginal benefit becomes $B^{\prime}(2 \bar{a}) \simeq c$. This implies the following optimal level of the uniform standard $\bar{a} \simeq \frac{1}{2} B^{\prime-1}(c)$, which is positive.

The preceding proof also provides the expression of the transfers when $\alpha \simeq 0: 2(1+\lambda) t \simeq B(2 \bar{a})-c \bar{a}(1-(1+\lambda) \delta)-B\left(B^{\prime-1}(c)\right)+c B^{\prime-1}(c)$.

If we use the assumption $\delta(1+\lambda) \simeq 1$ and introduce the expression of the optimal uniform standard, we obtain: $(1+\lambda) t \simeq \frac{1}{2} c B^{\prime-1}(c)$.

Let us return to the study of the levels of differentiated standards $a_{1}$ and $a_{2}$. 


$$
a_{1}=\bar{a}^{*}+\frac{(1+\lambda) t^{*}}{c} \Longleftrightarrow a_{1}=\frac{1}{2} B^{\prime-1}(c)+\frac{1}{2} B^{\prime-1}(c) \Longleftrightarrow a_{1}=B^{\prime-1}(c) \leq 1
$$

if $(c)$ is sufficiently large and $B^{\prime-1}($.$) is sufficiently small.$

$$
a_{2}=\bar{a}^{*}-\frac{(1+\lambda) t^{*}}{c} \Longleftrightarrow a_{2}=\frac{1}{2} B^{\prime-1}(c)-\frac{1}{2} B^{\prime-1}(c) \Longleftrightarrow a_{2}=0
$$

C3- Proof of Proposition 3

The maximum value attainable by the social welfare function in the UT agreement is:

$\bar{V}^{*}=\left[B\left(2 \bar{a}^{*}\right)-C\left(\bar{a}^{*}\right)-(1+\lambda) t^{*}-N B_{1}\right]^{\gamma} \times\left[\alpha B\left(2 \bar{a}^{*}\right)-\delta C\left(\bar{a}^{*}\right)+t^{*}-N \hat{B}_{2}\right]^{1-\gamma}$

This function, if $\alpha$ is strictly positive, can be written as follows:

$\frac{\bar{V}^{*}}{\alpha^{1-\gamma}}=\left[B\left(2 \bar{a}^{*}\right)-C\left(\bar{a}^{*}\right)-(1+\lambda) t^{*}-N B_{1}\right]^{\gamma} \times\left[B\left(2 \bar{a}^{*}\right)-\frac{\delta}{\alpha} C\left(\bar{a}^{*}\right)+\frac{t^{*}}{\alpha}-\frac{N B_{2}}{\alpha}\right]^{1-\gamma}$

Now we can write the social welfare function with particular values of the differentiated standards:

$$
\frac{V\left(a_{1}, a_{2}\right)}{\alpha^{1-\gamma}}=\left[B\left(a_{1}+a_{2}\right)-C\left(a_{1}\right)-N B_{1}\right]^{\gamma} \times\left[B\left(a_{1}+a_{2}\right)-\frac{\delta}{\alpha} C\left(a_{2}\right)-\frac{N B_{2}}{\alpha}\right]^{1-\gamma}
$$

Notice that the first terms in the brackets in Equations 15 and 16 are the same because of Definition 1. Hence, the condition of superiority of the differentiated social welfare function over the uniform becomes:

$$
\left[B\left(a_{1}+a_{2}\right)-\frac{\delta}{\alpha} C\left(a_{2}\right)-\frac{N B_{2}}{\alpha}\right]^{1-\gamma}>\left[B\left(2 \bar{a}^{*}\right)-\frac{\delta}{\alpha} C\left(\bar{a}^{*}\right)+\frac{t^{*}}{\alpha}-\frac{N B_{2}}{\alpha}\right]^{1-\gamma}
$$




$$
\Longleftrightarrow\left[-\delta C\left(a_{2}\right)\right]>\left[-\delta C\left(\bar{a}^{*}\right)+t^{*}\right]
$$

because $B\left(a_{1}+a_{2}\right)=B\left(2 \bar{a}^{*}\right)$ by Definition $1, \alpha$ and $(1-\gamma)$ are positive. From Definition 1, we have $t^{*}=\frac{C\left(a_{1}\right)-C\left(\bar{a}^{*}\right)}{1+\lambda}$. Introducing this expression of transfers into Equation 18, we obtain:

$$
\begin{gathered}
{\left[-\delta C\left(a_{2}\right)\right]>\left[-\delta C\left(\bar{a}^{*}\right)+\frac{C\left(a_{1}\right)-C\left(\bar{a}^{*}\right)}{1+\lambda}\right]} \\
\Longleftrightarrow \delta(1+\lambda)\left[C\left(\bar{a}^{*}\right)-C\left(a_{2}\right)\right]>\left[C\left(a_{1}\right)-C\left(\bar{a}^{*}\right)\right]
\end{gathered}
$$

From Condition C1 and the first condition of Definition 1, we have $a_{2}<\bar{a}^{*}<a_{1}$. This implies the positivity of the terms in brackets in Condition 2, say $\left[C\left(\bar{a}^{*}\right)-C\left(a_{2}\right)\right]>0$ and $\left[C\left(a_{1}\right)-C\left(\bar{a}^{*}\right)\right]>0$.

C4- Superiority of $a_{1}^{*}$ over $a_{2}^{*}$ (Condition C3)

Our objective here is to show that $a_{1}^{*}>a_{2}^{*}$, when the countries have the same bargaining powers $\gamma=1 / 2$, and a linear cost function $C(a)=c a$, where $c$ is a positive parameter.

The first-order conditions in the $\mathrm{D}$ agreement, when $\gamma=1 / 2$, are the following:

$$
\begin{aligned}
& \frac{\partial V}{\partial a_{1}}=0 \Longleftrightarrow \frac{\left[B^{\prime}\left(a_{1}+a_{2}\right)-c\right]}{\left[B\left(a_{1}+a_{2}\right)+\Omega_{1}-c a_{1}-\hat{N B_{1}}\right]}+\frac{\left[\alpha B^{\prime}\left(a_{1}+a_{2}\right)\right]}{\left[\alpha B\left(a_{1}+a_{2}\right)+\Omega_{2}-\delta c a_{2}-\hat{N B_{2}}\right]}=0 \\
& \frac{\partial V}{\partial a_{2}}=0 \Longleftrightarrow \frac{\left[B^{\prime}\left(a_{1}+a_{2}\right)\right]}{\left[B\left(a_{1}+a_{2}\right)+\Omega_{1}-c a_{1}-\hat{N B_{1}}\right]}+\frac{\left[\alpha B^{\prime}\left(a_{1}+a_{2}\right)-\delta c\right]}{\left[\alpha B\left(a_{1}+a_{2}\right)+\Omega_{2}-\delta c a_{2}-\hat{N B_{2}}\right]}=0
\end{aligned}
$$

The ratio of these first-order conditions gives us (assuming that we do not divide by 0$)$ :

$$
\frac{B^{\prime}\left(a_{1}+a_{2}\right)-c}{B^{\prime}\left(a_{1}+a_{2}\right)}=\frac{\alpha B^{\prime}\left(a_{1}+a_{2}\right)}{\alpha B^{\prime}\left(a_{1}+a_{2}\right)-\delta c}
$$




$$
\Longleftrightarrow B^{\prime}\left(a_{1}+a_{2}\right)=\frac{\delta c}{\delta+\alpha} \Longleftrightarrow a_{1}+a_{2}=B^{\prime-1}\left(\frac{\delta c}{\delta+\alpha}\right)
$$

If we replace $B^{\prime}\left(a_{1}+a_{2}\right)=\frac{\delta c}{\delta+\alpha}$ in Equation 21, we obtain the following relation: $U_{2}=\delta U_{1}$

$$
\text { where } U_{1}=\left[B\left(a_{1}+a_{2}\right)+\Omega_{1}-c a_{1}-\hat{N B_{1}}\right] \text { and } U_{2}=\left[\alpha B\left(a_{1}+a_{2}\right)+\Omega_{2}-\delta c a_{2}-\hat{N B_{2}}\right] \text {. }
$$

We thus have:

$$
\begin{aligned}
U_{2}=\delta U_{1} & \Longleftrightarrow\left[\alpha B\left(a_{1}+a_{2}\right)+\Omega_{2}-\delta c a_{2}-\hat{N B_{2}}\right]=\delta\left[B\left(a_{1}+a_{2}\right)+\Omega_{1}-c a_{1}-\hat{N B_{1}}\right] \\
& \Longleftrightarrow(\delta-\alpha) B\left(a_{1}+a_{2}\right)+\hat{N B_{2}}-\delta \hat{N B_{1}}=\delta c\left(a_{1}-a_{2}\right)
\end{aligned}
$$

We are interested in whether the term $(\delta-\alpha) B\left(a_{1}+a_{2}\right)+\hat{N B_{2}}-\delta \hat{N B} B_{1}$ is positive. We replace the expressions of the utility levels at the threat point $\hat{N B} B_{1}$ and $\hat{N B}$ which were calculated above, and obtain:

$$
(\delta-\alpha)\left[B\left(B^{\prime-1}\left(\frac{\delta c}{\delta+\alpha}\right)\right)-B\left(B^{\prime-1}(c)\right)\right]+\delta c B^{\prime-1}(c)=\delta c\left(a_{1}-a_{2}\right)
$$

We have $B^{\prime-1}\left(\frac{\delta c}{\delta+\alpha}\right)>B^{\prime-1}(c)$ because $\frac{\delta c}{\delta+\alpha}<c$ and $B^{\prime-1}($.$) is a decreasing$ function. We can conclude then that $a_{1}^{*}>a_{2}^{*}$ if $\delta>\alpha$.

\section{C5- Proof of Proposition 4}

The condition of superiority of the social welfare function in the UT agreement over the one in the $\mathrm{D}$ agreement $V^{*}\left(a_{1}^{*}, a_{2}^{*}\right)$ is:

$$
\begin{aligned}
\frac{\bar{V}(\bar{a}, t)}{\alpha^{1-\gamma}} & =\left[B(2 \bar{a})-C(\bar{a})-(1+\lambda) t-N \hat{B}_{1}\right]^{\gamma} \times\left[B(2 \bar{a})-\frac{\delta}{\alpha} C(\bar{a})+\frac{t}{\alpha}-\frac{N B_{2}}{\alpha}\right]^{1-\gamma} \\
\frac{V^{*}\left(a_{1}^{*}, a_{2}^{*}\right)}{\alpha^{1-\gamma}} & =\left[B\left(a_{1}^{*}+a_{2}^{*}\right)-C\left(a_{1}^{*}\right)-N B_{1}\right]^{\gamma} \times\left[B\left(a_{1}^{*}+a_{2}^{*}\right)-\frac{\delta}{\alpha} C\left(a_{2}^{*}\right)-\frac{N B_{2}}{\alpha}\right]^{1-\gamma}
\end{aligned}
$$


Notice that the first terms in the brackets in Equation 22 are the same because of Definition 2. Hence, the condition of superiority of the uniform social welfare function over the differentiated function becomes:

$$
[-\delta C(\bar{a})+t]>\left[-\delta C\left(a_{2}^{*}\right)\right]
$$

Given Definition 2, we have $t=\frac{C\left(a_{1}^{*}\right)-C(\bar{a})}{(1+\lambda)}$. By introducing this expression of the transfer in Equation 23, we obtain the result. Condition C3 and the first condition of Definition 2 imply the positivity of the terms in brackets in Condition C4, that is, $\left[C(\bar{a})-C\left(a_{2}^{*}\right)\right]>0$ and $\left[C\left(a_{1}^{*}\right)-C(\bar{a})\right]>0$.

\section{D- Individual Welfare}

\section{Proof of Proposition 5}

We begin the proof with a remark. Based on Proposition 2, we know that the social welfare in the UT agreement $\bar{V}^{*}$ is greater than that in the $\mathrm{D}$ agreement $V^{*}$, if the condition $\delta(1+\lambda)\left[C(\bar{a})-C\left(a_{2}^{*}\right)\right]<\left[C\left(a_{1}^{*}\right)-C(\bar{a})\right]$ (Condition C4) is verified, and if $a_{1}^{*}$ is superior to $a_{2}^{*}$ (Condition C3). This condition implies

the

relation $(1+\lambda) \delta \frac{C^{\prime}\left(a_{2}\right)}{C^{\prime}\left(a_{1}\right)}<1$ if $a_{1}^{*}$ is superior to $a_{2}^{*}$, because increasing marginal abatement costs lead to $\frac{\left[C\left(a_{1}^{*}\right)-C(\bar{a})\right]}{a_{1}^{*}-\bar{a}}<C^{\prime}\left(a_{1}^{*}\right)$ and $\frac{\left[C(\bar{a})-C\left(a_{2}^{*}\right)\right]}{\bar{a}-a_{2}^{*}}>C^{\prime}\left(a_{2}^{*}\right)$.

We use the first-order conditions of the programs associated with the UT and D agreements. We obtain:

$$
\begin{gathered}
(\mathrm{UT}) \gamma(1+\lambda)\left(N B_{2}^{U}-N \hat{B}_{2}\right)=(1-\gamma)\left(N B_{1}^{U}-N \hat{B}_{1}\right) \\
(\mathrm{D}) \gamma C^{\prime}\left(a_{1}^{*}\right)\left(N B_{2}^{D}-N B_{2}\right)=(1-\gamma) \delta C^{\prime}\left(a_{2}^{*}\right)\left(N B_{1}^{D}-N B_{1}\right)
\end{gathered}
$$

Proposition 2 implies that $\bar{V}^{*}$ is greater than $V^{*}$ under Conditions C3 and C4. So we have $\bar{V}^{*}=\left(\frac{\gamma(1+\lambda)}{(1-\gamma)}\right)^{\gamma}\left(N B_{2}^{U}-N B_{2}\right)>$ $\left(\frac{\gamma C^{\prime}\left(a_{1}^{*}\right)}{(1-\gamma) \delta C^{\prime}\left(a_{2}^{*}\right)}\right)^{\gamma}\left(N B_{2}^{D}-N B_{2}\right)=V^{*}$. As $(1+\lambda) \delta \frac{C^{\prime}\left(a_{2}^{*}\right)}{C^{\prime}\left(a_{1}^{*}\right)}<1, N B_{2}^{U}$ is greater than $N B_{2}^{D}$. Moreover $\left(N B_{1}^{U}-N B_{1}\right)>\left(N B_{1}^{D}-N B_{1}\right)$ if and only if $\frac{\gamma(1+\lambda)}{(1-\gamma)}\left(N B_{2}^{U}-N B_{2}\right)>\frac{\gamma C^{\prime}\left(a_{1}^{*}\right)}{(1-\gamma) \delta C^{\prime}\left(a_{2}^{*}\right)}\left(N B_{2}^{D}-N \hat{B}_{2}\right)$. 